\title{
Estudio de la transferencia de calor en la etapa de cocido en la elaboración de aceitunas verdes al estilo sevillano
}

\author{
Por M. Tarrado-Castellarnau ${ }^{\mathrm{a}}$, J.M. Domínguez Ortega ${ }^{\mathrm{b}}$, \\ A. Tarrado-Castellarnau ${ }^{c}$ y R. Pleite Gutiérrez ${ }^{b}$,
}

\author{
${ }^{a}$ Facultad de Biología, Departamento de Bioquímica y Biología Molecular, Universitat de Barcelona, \\ Av. Diagonal 643, 08028 Barcelona \\ ${ }^{b}$ Global Olive Consulting, c/ Contador 7, 41530 Morón de la Frontera, Sevilla \\ ${ }^{\circ}$ Departamento de Tecnología de Alimentos, Universitat de Lleida, \\ Av. Alcalde Rovira Roure 191, 25198 Lleida \\ Autor para la correspondencia: info@global-olive.es
}

\section{RESUMEN}

Estudio de la transferencia de calor en la etapa de cocido en la elaboración de aceitunas verdes al estilo sevillano

En este artículo se describe por primera vez a nivel industrial el aumento de temperatura que se produce en el interior de los tanques de elaboración durante la etapa de cocido. A partir del estudio de las características térmicas de la etapa de cocido se han podido determinar relaciones entre las variables que definen el proceso. Se ha demostrado que la temperatura al inicio del tratamiento de cocido influye en otras de las características del proceso como la duración de éste o la pendiente del aumento lineal de temperatura durante la etapa. El estudio establece que esta generación de calor puede provenir principalmente de las reacciones de hidrólisis alcalina que ocurren en el interior del fruto y, en menor proporción, de la dilución de la solución de hidróxido de sodio con el agua presente en la pulpa de las aceitunas.

PALABRAS CLAVE: Aceitunas de mesa - Cocido - Estilo sevillano - Hidrólisis - Temperatura - Transferencia de calor.

\section{SUMMARY}

Study of the heat transfer during the alkaline treatment in the processing of Spanish Style green table olives

This article describes for the first time at the industrial level the temperature rise that occurs inside the processing tank during the lye treatment. Relationships between variables that define the lye treatment have been determined from the study of the thermal characteristics of this process. The initial temperature influences other variables of this treatment, such as its duration or the slope of the linear increase of temperature produced. The study estates that this generation of heat can be principally caused by the alkaline hydrolysis reactions that occur in the interior of the fruit and, to a lesser extent, by the dilution of sodium hydroxide solution with water present in the pulp of the olives.

KEY-WORDS: Alkaline treatment - Heat transfer Hydrolysis - Table olives - Temperature - Spanish Style.

\section{INTRODUCCIÓN}

Las aceitunas de mesa verdes al estilo sevillano se elaboran a partir de variedades de aceitunas recolectadas en el estadio previo al envero y con la consistencia y tamaño deseados. El proceso está condicionado por dos etapas fundamentales, el cocido y la fermentación láctica en salmuera. La etapa de cocido consiste en el tratamiento de los frutos con una solución acuosa de hidróxido de sodio $(\mathrm{NaOH})$ al 2-4\% (p/v) durante unas 6-12 horas dependiendo de la variedad, el estado de maduración de los frutos y la temperatura de la solución. Una vez la difusión de la sosa ha llegado entre 2/3 y 3/4 de la pulpa, se detiene el tratamiento y se elimina la solución de $\mathrm{NaOH}$ y el exceso de ésta mediante uno o varios lavados estáticos con agua. Finalmente, se sumergen las aceitunas en una solución de salmuera a una concentración del 10-12\% (p/v) de cloruro de sodio donde se lleva a cabo la fermentación láctica para evitar la proliferación de microorganismos indeseables y promover el crecimiento de los lactobacilos (Fernández-Díez et al., 1985).

Los objetivos principales de la etapa de cocido son, por un lado, la eliminación del amargor natural que el glucósido oleuropeína confiere a las aceitunas y por otro lado, el aumento de la permeabilidad de los frutos para facilitar la salida de los metabolitos a partir de los cuales las bacterias lácticas presentes en la salmuera llevan a cabo la fermentación (Sánchez et al., 2006). Durante el tratamiento de cocido, el hidróxido de sodio hidroliza los enlaces éster de los componentes estructurales del epicarpio, solubilizando y eliminando gran parte de la capa cerosa que lo recubre y penetrando hacia el mesocarpio (Mafra et al., 2001). De este modo, se producen cambios estructurales tanto en la piel como en la pulpa, reduciendo el espesor de la cutícula y permeabilizando las diferentes capas de tejido. Todos estos cambios estructurales provocan la separación de las células y, en consecuencia, la pérdida de firmeza del fruto (Marsilio et al., 1996). Es- 
tas modificaciones dependen de la variedad de las aceitunas, la temperatura del tratamiento, la concentración de $\mathrm{NaOH}$ y la duración de la etapa de cocido.

Otros autores han estudiado la etapa de cocido a partir de la difusión de sodio (Zuritz y Maldonado, 2004; Maldonado y Zuritz, 2004a, 2004b; Maldonado et al., 2008 y Maldonado et al., 2011) y considerado su relación con la temperatura inicial y la concentración inicial de la solución de hidróxido de sodio (Maldonado et al., 2003; Zuritz et al. 2003; Maldonado y Zuritz, 2003a, 2003b). También se han publicado estudios en los que se propone trabajar con soluciones de cocido a baja temperatura e introducir una etapa de reposo con hidróxido de sodio de baja concentración para obtener productos finales de mayor calidad (Rejano et al., 2008; Carmona et al., 2011). Sin embargo, en ninguna de estas publicaciones se ha estudiado la evolución de la temperatura durante la etapa de cocido y sus efectos sobre los otros parámetros del proceso de cocido.

\subsection{Justificación y objetivos}

Las condiciones en que se desarrolla la etapa de cocido son determinantes en las etapas posteriores del proceso y en la calidad del producto final obtenido (Chammem et al., 2005).

Al tratarse el cocido de un proceso de elaboración tradicional con metodologías variables en función de la zona geográfica donde se realiza, hasta la fecha no se han fijado unas condiciones de trabajo homogéneas. Asimismo, la mayor parte de la investigación realizada hasta el momento se ha centrado en el estudio de la etapa de fermentación, común en otros vegetales (como pepinillos, por ejemplo). La etapa de cocido, típica de la aceituna, no ha recibido tanta atención por parte de la comunidad científica.

Es por todo ello que la caracterización de la etapa de cocido en la elaboración de aceitunas verdes al estilo sevillano resulta de especial interés.

El objetivo del presente trabajo es la caracterización de la transferencia de calor en el proceso de cocido en la elaboración de aceitunas verdes al estilo sevillano, mediante el estudio de la evolución de la temperatura durante este tratamiento y partiendo de datos obtenidos experimentalmente de un proceso industrial.

En este estudio se partirá de datos reales tomados de las operaciones habituales de trabajo en plantas industriales de elaboración de aceitunas verdes y se pretende que los resultados puedan aplicarse en la optimización industrial de la etapa de cocido.

\section{MATERIALES Y MÉTODOS}

\subsection{Material vegetal}

Para este estudio se han utilizado aceitunas de la variedad hojiblanca, procesadas durante la cam- paña 2010-11 en la empresa Nuestra Señora de las Virtudes S.C.A. de la Puebla de Cazalla (SeviIla, España), y aceitunas de la variedad picual procesadas durante la campaña 2011 en la empresa Víctor Giordana S.A. de Vichigasta (La Rioja, Argentina). Se han estudiado paralelamente los datos procedentes de estos dos sistemas de producción diferentes para tener unos resultados más completos y consistentes.

\subsection{Sistema de elaboración}

En ambas campañas los tanques utilizados para el cocido de aceitunas fueron depósitos de fibra de vidrio y poliéster, de forma cónica en los extremos y cilíndrica en la parte central, de una capacidad de 16.000 litros $(10.000 \mathrm{~kg}$ de aceitunas y 6.000 I de sosa de capacidad, aproximadamente).

Los depósitos de cocido en la campaña argentina estaban ubicados a la intemperie en el exterior de la industria, mientras que en la campaña española los depósitos de cocido estaban en el interior de la industria. En ambos casos los procesos de cocido tuvieron lugar durante la noche.

La concentración de la sosa empleada para el cocido en ambas campañas fue de 3,0 $\pm 0,2 \%$ (p/v).

\subsection{Toma de muestras}

Durante el cocido de las aceitunas se han tomado muestras con una frecuencia aproximada de 1 hora. Para este muestreo se ha utilizado un tubo de PVC que permite obtener muestras de líquido y aceitunas localizadas en cualquier punto de la altura del depósito de cocido. En cada muestreo se han tomado unas 30 aceitunas de un tamaño similar para el seguimiento del proceso de cocido y para la medida de la temperatura interna de la aceituna.

Durante el proceso de cocido, se ha tomado la temperatura de la solución de hidróxido de sodio cada minuto por medio de una sonda de temperatura colocada en el interior de cada depósito. Asimismo, se ha tomado cada hora la temperatura interior de 20 aceitunas mediante la introducción de una sonda de temperatura en los frutos.

\subsection{Equipos}

Para la medida de temperatura se han usado sondas digitales de la marca DALLAS SEMICONDUCTOR calibrados de fábrica (error máximo $0,5^{\circ} \mathrm{C}$ y resolución $1 / 16^{\circ} \mathrm{C}$ ). Cada sonda está asociada a una tarjeta de adquisición que transforma la señal a un protocolo RS232. Esta información es procesada por el software SCADA que informa de forma gráfica de la evolución de la temperatura. La instalación industrial tipo consta de una sonda exterior para medir la temperatura ambiente y una sonda de temperatura en el interior de cada uno de los depósitos de cocido. 
Para el control y registro de datos se ha desarrollado un software para PC en Visual Basic 6.0, capaz de registrar la información procedente de los sensores de temperatura, generar una base de datos y representar gráficamente los resultados.

\subsection{Modelo matemático para el estudio de la transmisión de calor en la etapa de cocido de la aceituna}

Todo el tratamiento matemático se ha realizado sobre el modelo de trabajo teórico formado por una única aceituna y rodeada por el volumen de solución de $\mathrm{NaOH}$ que le corresponde. En este caso, teniendo en cuenta un peso medio de las aceitunas de 8,6 gramos (Al-Widyan et al., 2010), el volumen de sosa por aceituna es de 5,16 mililitros. En la Figura 1 se encuentra representado el modelo de trabajo teórico con los valores de longitud y volumen que lo describen. Se utilizan datos bibliográficos de peso y dimensión de aceituna verde para poder diseñar un modelo matemático general que permita englobar todas las variedades de aceituna verde.

Para la descripción del modelo se realizan las siguientes consideraciones teóricas:

1. La pulpa de la aceituna es una esfera de radio interno $r_{i}\left(r_{i}=6 \mathrm{~mm}\right)$ y radio externo $r_{0}$ $\left(r_{0}=13,19 \mathrm{~mm}\right)$ rodeada por una epidermis de radio interno $r_{0}$ y radio externo $r_{s}$ $\left(r_{s}=13,23 \mathrm{~mm}\right)$.

2. El hueso y la capa de pulpa que lo recubre y que no llega a ser atacada por la solución de hidróxido de sodio forman una esfera sólida de radio $r_{i}$ concéntrica a la aceituna (representada en la Figura 1 por la línea de puntos).

Para determinar la transmisión de calor entre la aceituna que se encuentra a una temperatura $T_{s, 0} y$ la solución de hidróxido de sodio a una temperatura $T_{0,1}$ durante la etapa de cocido, se tiene que tener en cuenta que esta transferencia de calor es en estado no estacionario, de manera que la ecuación que define este proceso es la siguiente:

$$
\frac{\partial T_{s}}{\partial t}=\alpha\left(\frac{\partial^{2} T_{s}}{\partial x^{2}}+\frac{\partial^{2} T_{s}}{\partial y^{2}}+\frac{\partial^{2} T_{s}}{\partial z^{2}}\right)
$$

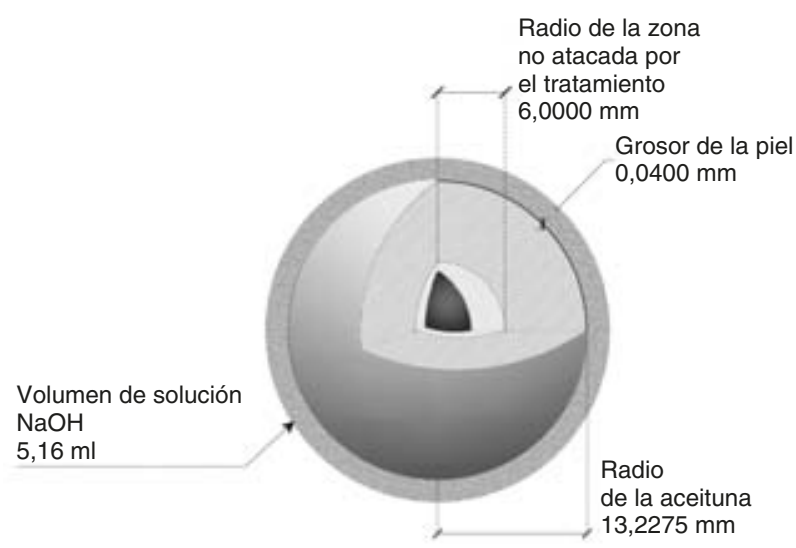

Figura 1

Representación del modelo de trabajo teórico.
Donde $T_{\mathrm{s}}$ es la temperatura en un punto cualquiera de la aceituna, y $\alpha$ la difusividad térmica $\left[\mathrm{m}^{2} / \mathrm{s}\right]$ que únicamente depende de la naturaleza y el estado del sólido y viene definida por:

$$
\alpha=\frac{\mathrm{k}_{\mathrm{s}}}{\rho_{\mathrm{s}} \mathrm{C}_{\mathrm{p}, \mathrm{s}}}
$$

Donde $\mathrm{k}_{\mathrm{s}}$ es la conductividad, $\rho_{\mathrm{s}}$ la densidad y $\mathrm{C}_{\mathrm{p}, \mathrm{s}}$ el calor específico del sólido.

El balance microscópico de energía es:

$$
\frac{\partial}{\partial \mathrm{t}}\left(\rho_{\mathrm{s}} \mathrm{C}_{\mathrm{p}, \mathrm{s}} \mathrm{T}\right)=-\nabla \mathrm{q}
$$

Aplicando la Ley de Fourier $\left(\mathrm{q}=-\mathrm{k}_{\mathrm{s}} \nabla \mathrm{T}\right.$ [2.4]) y suponiendo que tanto la densidad $\left(\rho_{\mathrm{s}}\right)$ como el calor específico $\left(\mathrm{C}_{\mathrm{p}, \mathrm{s}}\right)$ y la conductividad del sólido $\left(\mathrm{k}_{\mathrm{s}}\right)$ son constantes, se obtiene:

$$
\frac{\partial \mathrm{T}}{\partial \mathrm{t}}=\alpha \nabla^{2} \mathrm{~T}
$$

Trabajando en coordenadas esféricas y determinando que la transmisión de calor se da en la dirección radial:

$$
\nabla^{2}=\frac{1}{r^{2}} \frac{\partial}{\partial r}\left(r^{2} \frac{\partial}{\partial r}\right)=\frac{2}{r} \frac{\partial}{\partial r}+\frac{\partial^{2}}{\partial r^{2}}
$$

Si se introduce en la ecuación [2.5], se obtiene la siguiente ecuación de balance de energía en estado no estacionario en la dirección radial:

$$
\frac{\partial \mathrm{T}}{\partial \mathrm{t}}=\alpha\left(\frac{\partial^{2} \mathrm{~T}}{\partial \mathrm{r}^{2}}+\frac{2}{\mathrm{r}} \frac{\partial \mathrm{T}}{\partial \mathrm{r}}\right)
$$

Para poder resolverla se determinan las siguientes condiciones límite (Costa et al., 2002):

- CL1: En el instante inicial, todos los puntos de la aceituna se encuentran a la misma temperatura:

$$
\mathrm{t}=0 \quad 0<\mathrm{r}<\mathrm{r}_{0} \quad \mathrm{~T}=\mathrm{T}_{\mathrm{s}, 0}
$$

- CL2: En el centro del sistema, la temperatura presenta en todo instante un máximo:

$$
\mathrm{t}>0 \quad \mathrm{r}=0 \quad \partial \mathrm{T} / \partial \mathrm{r}=0
$$

- CL3: En la superficie del sistema, la cantidad de calor que, en este caso, sale por convección desde la aceituna al exterior es igual a la que se transmite por conducción desde el interior:

$$
t>0 \quad r=r_{0} \quad h\left(T_{1}-T_{s}\right)=-k(\partial T / \partial r)
$$

Una medida adimensional para la conducción de calor, que viene dada por el tiempo de enfriamiento y el tamaño del objeto, se define por el número de Fourier:

$$
\mathrm{Fo}=\frac{\alpha \mathrm{t}}{\mathrm{L}^{2}}=\frac{\alpha \mathrm{t}}{(\mathrm{V} / \mathrm{A})^{2}}=\frac{\mathrm{k}_{\mathrm{s}}}{\rho_{\mathrm{s}} \mathrm{C}_{\mathrm{s}}} \frac{\mathrm{t}}{(\mathrm{V} / \mathrm{A})^{2}}
$$


La importancia relativa de los términos de resistencia en la superficie y en el interior se miden mediante el número de Biot, un grupo adimensional definido por:

$$
B i=\left(\begin{array}{c}
\begin{array}{c}
\text { resistencia } \\
\text { interior }
\end{array} \\
\begin{array}{c}
\text { resistencia } \\
\text { en la superficie }
\end{array}
\end{array}\right)=\frac{\mathrm{hL}}{\mathrm{k}_{\mathrm{s}}}=\frac{\mathrm{h}(\mathrm{V} / \mathrm{A})}{\mathrm{k}_{\mathrm{s}}}
$$

Para valores del número de Biot pequeños $(B i<0,1)$ la principal resistencia se encuentra en la superficie, mientras que para valores altos $(B i>40)$ toda la resistencia está definida por la conducción de calor hacia fuera del objeto y la resistencia de la superficie es despreciable (Levenspiel, 1998).

Si se considera que toda la resistencia se encuentra en la superficie del objeto, de manera que éste es isotermo en cualquier momento y punto del objeto, el balance de calor es el siguiente:

$$
-q=h A\left(T_{s}-T_{l}\right)=W C_{s} \frac{\partial T_{s}}{\partial t}
$$

Separando e integrando para $\frac{h A}{V_{\rho s} C_{p}}$ constante,
obtiene:

$$
\frac{\Delta T}{\Delta T_{\text {máx }}}=\frac{T_{s}-T_{1}}{T_{s, 0}-T_{1}}=e^{-F o \cdot B i}=e^{-\left(h_{t} / L_{\rho s} C_{p}\right)}
$$

Combinando las ecuaciones [2.10] y [2.11], se obtiene el caudal instantáneo de pérdida de calor desde el objeto:

$$
-q=-\rho_{s} C_{s} v \frac{\partial T_{s}}{\partial t}=h A\left(T_{s, o}-T_{1}\right) e^{-F o \cdot B i}
$$

Se ha comprobado que la resistencia a la transmisión de calor en la superficie de la aceituna controla el proceso, siendo uniforme la temperatura en toda la pulpa, mediante el programa informático HTTonedt de la Universidad de Virginia (EUA) (datos no mostrados).

Para poder describir los procesos de conducción de calor en la piel y de convección en la película alrededor del epicarpio, se define el coeficiente efectivo de convección $h_{e}$ que engloba las contribuciones de las dos a la resistencia en la superficie (Figura 2).

Conducción en la piel:

$$
\mathrm{q}=\mathrm{k} \frac{\Delta \mathrm{T}_{1}}{\Delta \mathrm{x}}
$$

Convección en la película: $\quad \mathrm{q}=\mathrm{h} \Delta \mathrm{T}_{2}$

Efecto global de la superficie: $\mathrm{q}=\mathrm{h}_{\mathrm{e}} \Delta \mathrm{T}_{\text {Total }}$

En la Figura 3 se representan los perfiles de temperatura en el inicio del proceso de transferencia de calor y en el final del tratamiento de cocido. Todo el proceso se desarrolla en estado no estacionario.

Para el estudio del proceso de dilución de la solución de hidróxido de sodio se parte del balance de materia del sistema esquematizado en la Figura 4.

La concentración final de $\mathrm{NaOH}$ de la solución al final del tratamiento de cocido se encuentra según:

$$
\mathrm{C}_{\mathrm{NaOH}, \mathrm{f}}=\frac{\mathrm{V}_{\mathrm{NaOH}} \cdot \mathrm{C}_{\mathrm{NaOH}, \mathrm{O}}}{\mathrm{V}_{\mathrm{H} 20}+\mathrm{V}_{\mathrm{NaOH}}}
$$

Teniendo en cuenta que $V_{\text {H2O }}$ es el volumen de agua y corresponde a la que se encuentra presente en las aceitunas considerando que el $60 \%$ de la composición de éstas es agua (Al-Widyan y col., 2008).
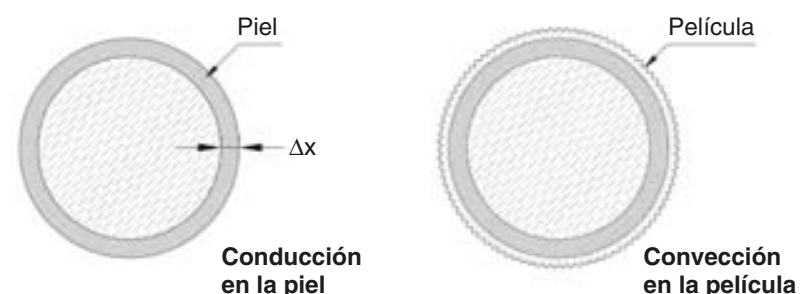

Figura 2

Representación de las capas implicadas en la transmisión de calor. (a) En la piel, la transferencia de calor se produce por conducción y depende del grosor $(\Delta \mathrm{x}$ ) de ésta. (b) En la película que recubre la piel, el proceso de intercambio de calor se da por convección.

Tabla 1

Propiedades geométricas y térmicas de la aceituna

\begin{tabular}{lclc}
\hline \multicolumn{2}{c}{ Propiedades geométricas aceituna } & \multicolumn{2}{c}{ Propiedades térmicas aceituna } \\
\hline Radio interno pulpa, $\mathrm{r}_{\mathrm{i}}(\mathrm{cm})$ & 0,6 & Conductividad, $\mathrm{k}_{\mathrm{s}}\left(\frac{\mathrm{w}}{\mathrm{mK}}\right)$ & 2,62 \\
Radio externo pulpa, $\mathrm{r}_{\mathrm{o}}(\mathrm{cm})$ & 1,319 & Densidad, $\rho_{\mathrm{s}}\left(\frac{\mathrm{kg}}{\mathrm{m}^{2}}\right)$ & 1101,4 \\
Radio externo piel, $\mathrm{r}_{\mathrm{s}}(\mathrm{cm})$ & 1,323 & Calor específico, $\mathrm{C}_{\mathrm{p}, \mathrm{s}}\left(\frac{\mathrm{J}}{\mathrm{kgK}}\right)$ & 3275 \\
& & & \\
\hline
\end{tabular}

Fuente: Al-Widyan et al., 2010. 

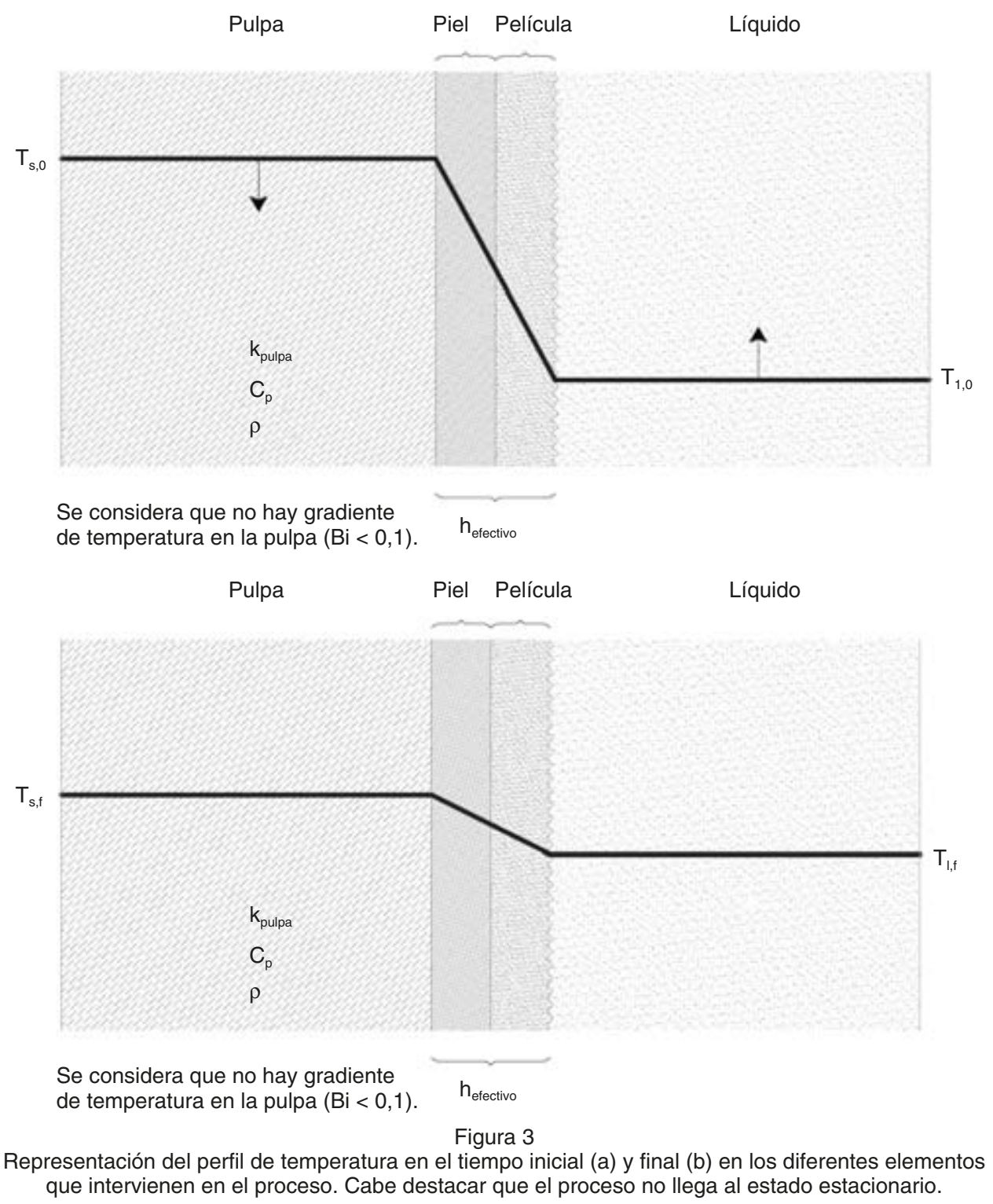

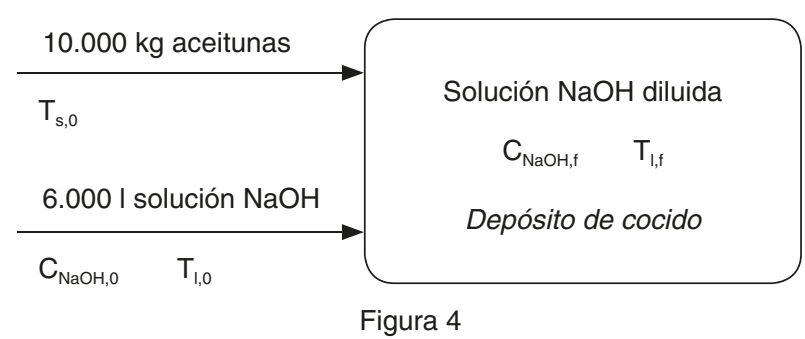

Esquema del balance de materia en el depósito de cocido.

Para calcular el aumento de temperatura que supone la dilución de la concentración de hidróxido de sodio se trabaja con las entalpías y los calores específicos de las disoluciones de sodio a las diferentes concentraciones y temperaturas de trabajo interpolando a partir de los valores que se encuentran tabulados en la bibliografía (Ficha de características del producto $\mathrm{NaOH}$ de Solvay Chemicals International, Tabla 2) y calculando:

- La entalpía de la solución de $\mathrm{NaOH}$ a la temperatura y la concentración inicial:

$$
\mathrm{H}_{\mathrm{NaCH}, 0}[\mathrm{~kJ}]=\mathrm{H}_{\mathrm{NaOH}, 0}\left[\frac{\mathrm{kJ}}{\mathrm{kg}}\right] \cdot \mathrm{r}_{\mathrm{NaOH}}\left[\frac{\mathrm{kg}}{\mathrm{l}}\right] \cdot \mathrm{V}_{\mathrm{NaOH}}[\mathrm{l}]
$$

Se toma la densidad de la sosa a la temperatura y concentración de trabajo como $1,03 \mathrm{~kg} / \mathrm{l}$ (Caustic Soda Solution Handbook, The Dow Chemical Company).

- La entalpía del agua presente dentro las aceitunas a la temperatura y la concentración inicial:

$$
\mathrm{H}_{\mathrm{H} 2 \mathrm{O}, \mathrm{O}}[\mathrm{kJ}]=\mathrm{H}_{\mathrm{H} 20,0}\left[\frac{\mathrm{kJ}}{\mathrm{kg}}\right] \cdot \mathrm{r}_{\mathrm{H} 2 \mathrm{O}}\left[\frac{\mathrm{kg}}{\mathrm{l}}\right] \cdot \mathrm{V}_{\mathrm{H} 2 \mathrm{O}}[\mathrm{I}]
$$

Se toma la densidad del agua a la temperatura y concentración de trabajo como 1,0 kg/l.

- La entalpía de la masa total de solución de $\mathrm{NaOH}$ diluida a la temperatura inicial de la solución de $\mathrm{NaOH}$ y la concentración final:

$$
-\mathrm{H}_{\text {NaOH,dil }}[\mathrm{kJ}]=-\mathrm{H}_{\text {NaOJ,ail }}\left[\frac{\mathrm{kJ}}{\mathrm{kg}}\right] \cdot\left(\mathrm{m}_{\text {NaOH }}+\mathrm{m}_{\text {H20 }}\right)[\mathrm{kg}]
$$


Tabla 2

Entalpías y calores específicos de las soluciones acuosas de hidróxido de sodio según su temperatura y concentración

\begin{tabular}{|c|c|c|c|c|c|c|}
\hline \multirow{3}{*}{$\begin{array}{c}\text { Concentración } \\
\text { [kg NaOH/kg solución] }\end{array}$} & \multirow{2}{*}{\multicolumn{3}{|c|}{$\begin{array}{c}\text { Entalpía [kJ/kg] } \\
\text { Temperatura }\left[{ }^{\circ} \mathrm{C}\right]\end{array}$}} & \multirow{2}{*}{\multicolumn{3}{|c|}{$\begin{array}{c}\text { Calor específico } \\
{\left[\left(\mathbf{k J} /\left(\mathrm{kg}^{\circ} \mathrm{C}\right)\right]\right.}\end{array}$}} \\
\hline & & & & & & \\
\hline & 10 & 20 & 30 & 10 & 20 & 30 \\
\hline 0 & 41,8 & 84,6 & 126,8 & 4,190 & 4,181 & 4,177 \\
\hline 0,02 & 41,9 & 82,3 & 123,0 & 4,053 & 4,063 & 4,072 \\
\hline 0,04 & 41,1 & 79,7 & 119,2 & 3,947 & 3,969 & 3,985 \\
\hline 0,06 & 39,7 & 76,9 & 115,5 & 3,867 & 3,894 & 3,915 \\
\hline
\end{tabular}

Fuente: Ficha de características de producto Solvay Chemicals International.

- El calor liberado $\left(q_{\text {liberado }}\right)$ :

$$
-\mathrm{H}_{\mathrm{NaOH}, \text { dil }}[\mathrm{kJ}]=-\mathrm{H}_{\mathrm{NaOJ}, \mathrm{dil}}\left[\frac{\mathrm{kJ}}{\mathrm{kg}}\right] \cdot\left(\mathrm{m}_{\mathrm{NaOH}}+\mathrm{m}_{\mathrm{H} 2 \mathrm{O}}\right)[\mathrm{kg}]
$$

- Finalmente, el aumento de temperatura resultante de la dilución de la solución de hidróxido de sodio $(\Delta T)$ :

$$
\Delta \mathrm{T}_{\text {dilución }}\left[{ }^{\circ} \mathrm{C}\right]=\frac{\mathrm{q}_{\text {liberado }}[\mathrm{kJ}]}{\mathrm{m}_{\text {total }}[\mathrm{kg}] \cdot \mathrm{C}_{\mathrm{e} \text { NaOH diluida }}\left[\frac{\mathrm{kJ}}{\mathrm{kg} \cdot{ }^{\circ} \mathrm{C}}\right]}
$$

\subsection{Análisis estadístico}

Mediante un análisis multivariante de los datos obtenidos se calculan los coeficientes de correla- ción de Pearson para determinar si existen relaciones lineales entre las variables estudiadas. Este análisis estadístico se ha realizado con el software informático Statgraphics Centurion XVI.

\section{RESULTADOS}

\subsection{Estudio de los perfiles de temperatura y caracterización de las variables principales durante la etapa de cocido}

La representación gráfica del perfil tipo de temperaturas en la etapa de cocido y en el lavado con agua posterior se encuentra en la Figura 5. Se distinguen tres etapas diferenciadas; la fase inicial en la cual la temperatura disminuye drásticamente debido a la adición de solución de $\mathrm{NaOH}$ que se en-

Evolución de la temperatura de la mezcla de solución de hidróxido sódico y aceitunas

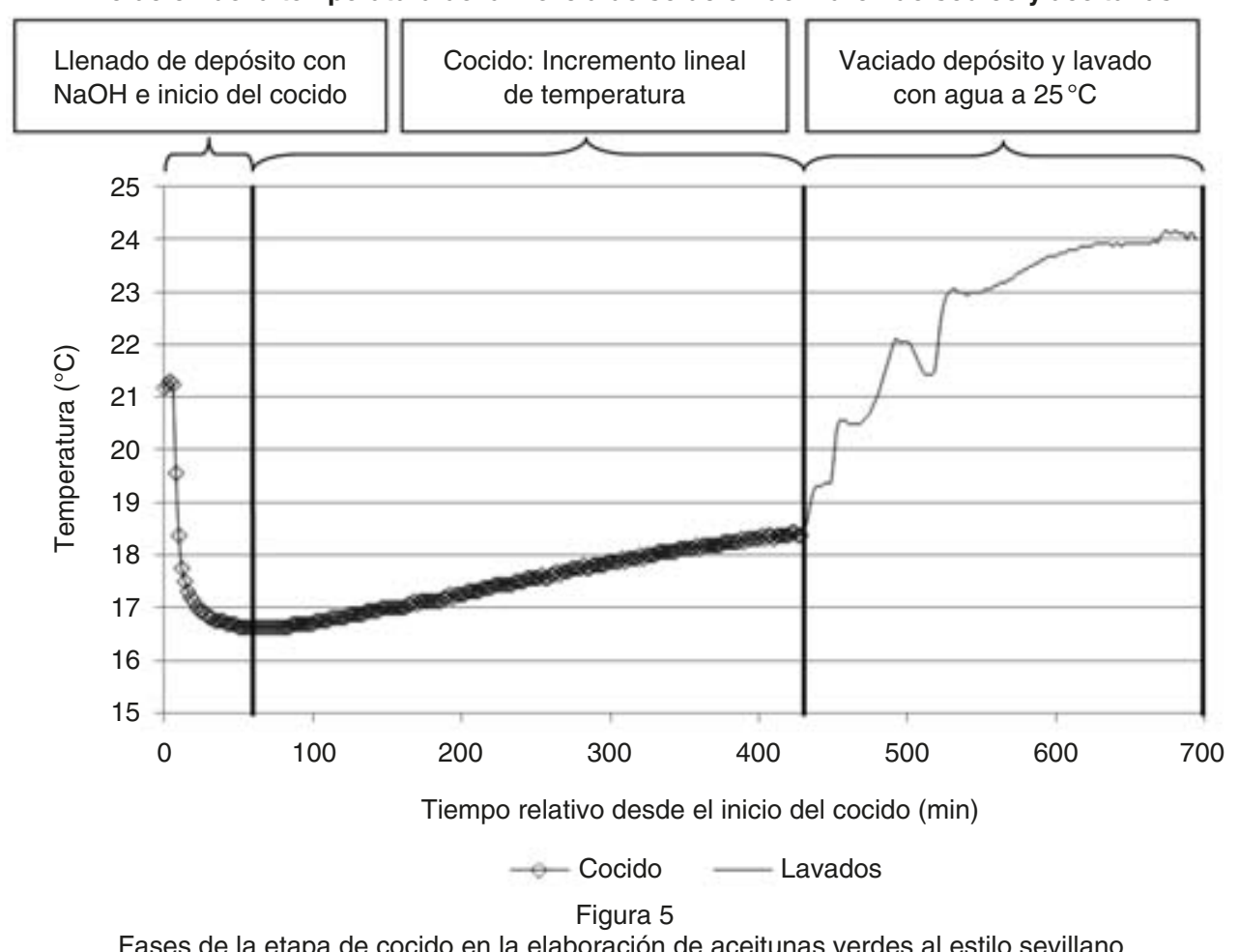

Fases de la etapa de cocido en la elaboración de aceitunas verdes al estilo sevillano. 
cuentra aproximadamente a $10^{\circ} \mathrm{C}$ sobre las aceitunas (cuya temperatura inicial ronda los $20^{\circ} \mathrm{C}$ ), la etapa de cocido con un aumento lineal de temperatura y el vaciado del depósito de cocido seguido del lavado de las aceitunas con agua a unos $25^{\circ} \mathrm{C}$ para la eliminación de la sosa. En la Figura 6 se representa el perfil de temperaturas en la etapa de cocido de tres procesos independientes por campaña. En todos los casos, se observa que durante el tratamiento de cocido la temperatura aumenta linealmente. El registro de la temperatura ambiental permite descartar la posibilidad que el aumento lineal de temperatura observado durante el cocido sea debido al intercambio de calor con el exterior. La representación del aumento lineal de temperatura con el tiempo durante el tratamiento de cocido permite la obtención de la información descriptiva de este proceso como la temperatura inicial de la solución de hidróxido de sodio, el incremento de temperatura que se produce, la pendiente de este aumento lineal de temperatura y la duración del cocido.

Se ha estudiado el perfil de temperaturas de dos grupos de experimentos, los correspondientes a la campaña 2011 de Argentina $(n=60)$ y los de la campaña 2010-11 de España $(n=35)$. En ambos casos los datos siguen la misma tendencia aunque haya diferencias significativas $(p>0,00001)$ entre las campañas en los rangos de valores absolutos. Era de esperar que existieran diferencias entre los valores de las dos campañas, ya que se trabaja con variedades distintas (Hojiblanca y Picual) y las condiciones de elaboración también han sido diferentes. Sin embargo, el estudio de las dos campañas en paralelo permite comparar si los datos obtenidos siguen la misma tendencia y si se observa un aumento lineal de temperatura durante el cocido en ambos casos. De este modo, se podría extrapolar este estudio a diferentes variedades como la manzanilla (ampliamente cultivada en España) y a otras regiones de cultivo con climas distintos.

Se representan gráficamente los resultados experimentales para mostrar la influencia de la temperatura de la mezcla de solución de hidróxido de sodio y aceitunas al inicio del cocido sobre la duración del tratamiento (Figura 7.A) y sobre la pendiente del aumento lineal de la temperatura (Figura 7.B) para concentraciones similares de la solución de hidróxido de sodio.

En las representaciones gráficas se puede extraer la misma conclusión, la temperatura de la mezcla al inicio del tratamiento influye en las otras características del proceso. Empezar la etapa de cocido con la mezcla de solución de hidróxido de sodio y aceitunas a una temperatura baja provoca un alargamiento del tiempo necesario para alcanzar el mismo grado de cocido y el aumento neto de temperatura es más bajo. Ahora bien, los tratamientos que se inician con una temperatura de la mezcla más elevada reducen su duración ya que la difusión de la sosa es más rápida y también se consigue un incremento de temperatura ligeramente superior. Hay que remarcar que hay otras variables a tener en cuenta a la hora de diseñar las condiciones de trabajo de la etapa, como por ejemplo que una temperatura suficientemente elevada durante el cocido puede favorecer la acción de las oxidasas y provocar daños en los frutos (Kader, 2011).

Los coeficientes de correlación de Pearson determinan la existencia de una relación lineal entre la variable de temperatura inicial de la mezcla de aceituna y solución de cocido y la de duración de tratamiento con un valor $p=0,00001$ para los datos de la campaña de Argentina y $p=0,0008$ para los de la campaña de España. De igual modo, también existe una relación lineal entre las variables de temperatura inicial de la mezcla y de pendiente del aumento lineal de temperatura con un valor $p=0,0001$ para los datos de la campaña de Argentina y $p=0,05$ para los de la campaña de España.

\subsection{Estudio de la transferencia de calor en la etapa de cocido}

En el tratamiento de cocido se ha utilizado una solución de hidróxido de sodio fría (a unos 10$15^{\circ} \mathrm{C}$ ) para minimizar los daños sobre las aceitu-

B

Evolución de la temperatura de la solución de $\mathrm{NaOH}$ (Campaña Argentina)

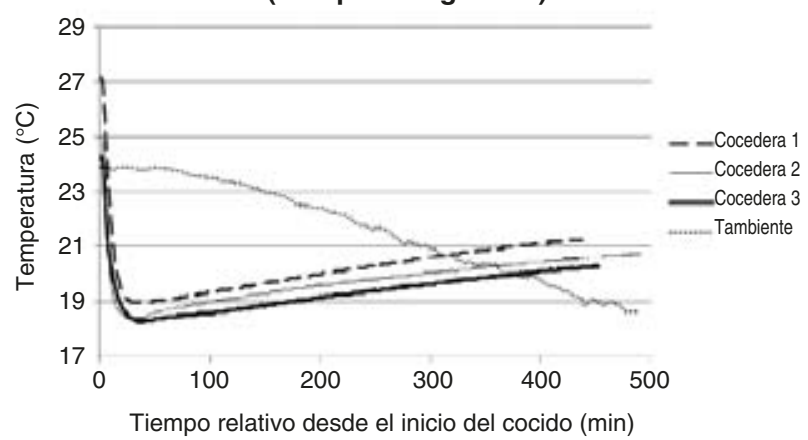

Figura 6

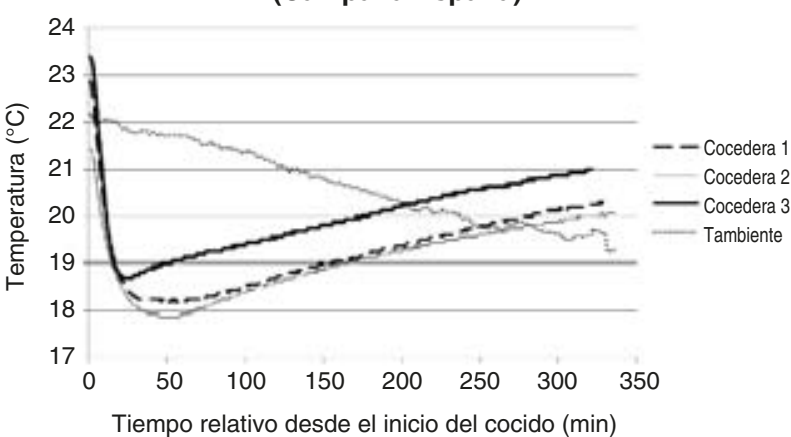

Evolución de la temperatura de la mezcla de solución de hidróxido de sodio y aceitunas en el interior del depósito de cocido. Se representan los datos obtenidos de tres procesos de cocido realizados en paralelo para la campaña española (A) y para la campaña argentina (B). 
A

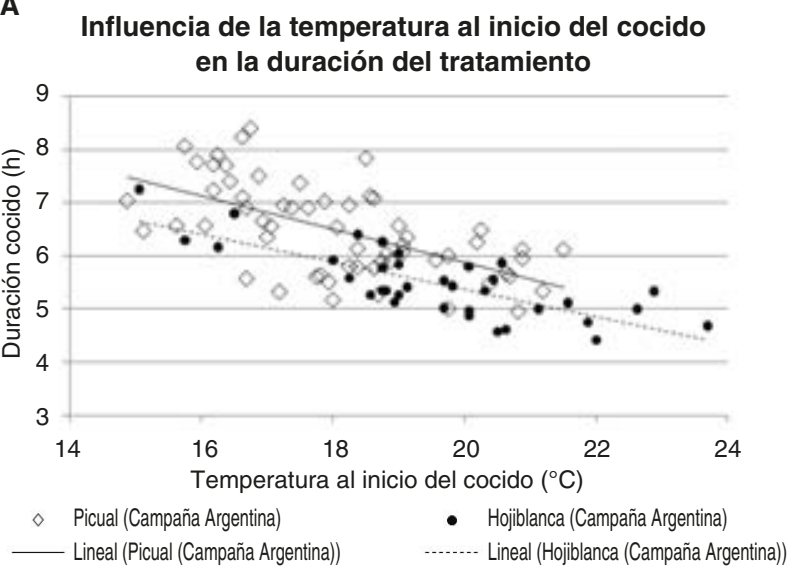

B

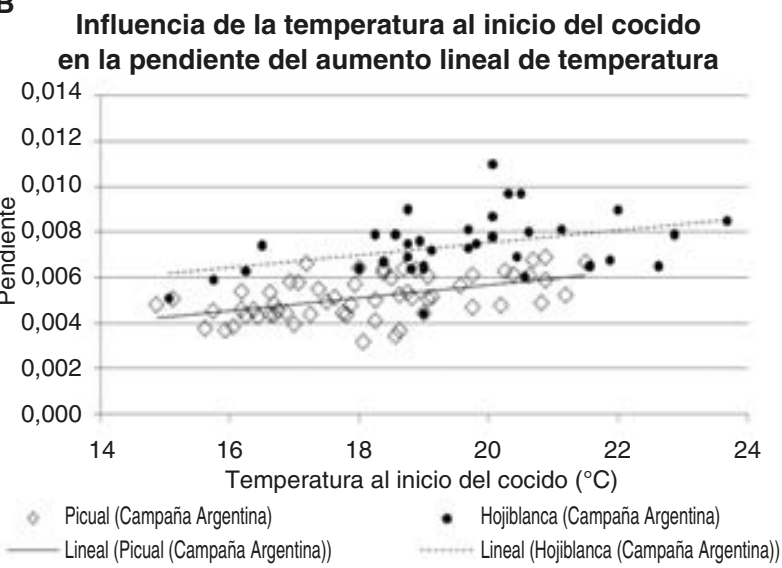

Figura 7

A. Representación de la influencia de la temperatura de la mezcla de solución de hidróxido de sodio y aceitunas al inicio del cocido sobre la duración del tratamiento. B. Representación de la influencia de la temperatura de la mezcla de solución de hidróxido de sodio y aceitunas al inicio del cocido sobre el valor de la pendiente del aumento lineal de temperatura durante el tratamiento. Cada punto representa un experimento independiente con la misma cantidad de aceitunas.

nas, ralentizando así la actividad de las enzimas oxidasa causantes del deterioro de los frutos, cuya temperatura óptima de reacción es superior (Kader, 2011).

Las aceitunas se encuentran a una temperatura más elevada $\left(20-25^{\circ} \mathrm{C}\right)$ en el interior del depósito y, una vez éste se llena con la solución fría de sosa y se estabiliza a un mínimo la temperatura marcada por la sonda del interior, empieza la etapa de transmisión de calor entre la solución y el interior de la aceituna correspondiéndose al inicio del cocido. No se ha considerado el intercambio de calor del depósito con el exterior para simplificar el modelo y porque se ha descartado que pueda ser responsable del aumento de temperatura lineal observado durante el cocido (Figura 6).

Para estudiar esta transferencia de calor se necesita conocer las propiedades geométricas y térmicas de la aceituna, recogidas en la Tabla 1.

Mediante estos datos se calculan los valores de los parámetros y las variables que definen el proceso de transferencia de calor obteniendo la temperatura teórica de la aceituna $\left(T_{s}\right)$ (Figura 8).

Una posible estimación para determinar la causa del aumento de la temperatura lineal durante la etapa de cocido es considerar que la dilución que se produce en la concentración de sosa con el agua tiene un papel en este incremento de temperatura. Así, teniendo en cuenta las temperaturas iniciales de los dos componentes protagonistas de la dilución (el agua a la temperatura interna de la aceituna y la solución inicial de $\mathrm{NaOH}$ ) y, por tanto, sus entalpías y la concentración final con el calor específico correspondiente, se puede hacer una estimación de la temperatura que alcanzaría la solución diluida resultante de la mezcla de los volúmenes de agua y solución de $\mathrm{NaOH}$ concentrada correspondientes.

Para calcular el aumento de temperatura teórico que conlleva esta dilución se trabaja con las entalpías y los calores específicos de las disoluciones de sodio de cada experimento, a unas concentracio- nes y temperaturas de trabajo diferentes, interpolando a partir de los valores de la Tabla 2.

Para estimar el calor liberado por la dilución y el aumento de temperatura resultante, se calcula la entalpía de la solución de $\mathrm{NaOH}$ y del agua presente dentro de las aceitunas a la temperatura y la concentración iniciales, y la entalpía de la masa total de solución de $\mathrm{NaOH}$ diluida a la temperatura inicial de la solución de $\mathrm{NaOH}$ y a la concentración final.

Comparando los resultados obtenidos, se puede observar que la media del incremento de temperatura calculado es inferior al real medido experimentalmente (datos no mostrados). De este modo, aunque se aceptara la suposición de que el agua

\section{Evolución de la temperatura durante la etapa de cocido}

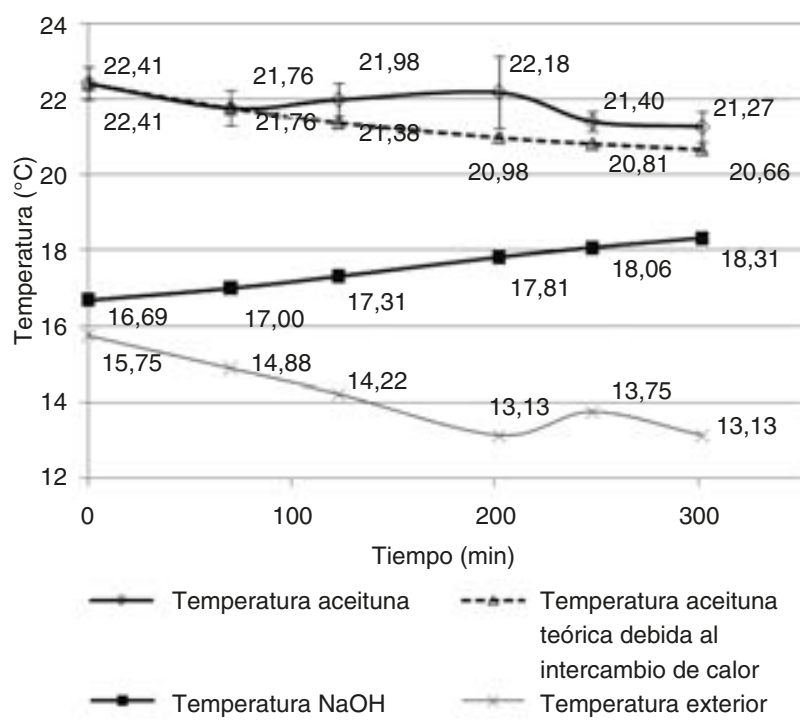

Figura 8

Representación de la evolución de la temperatura interior de la aceituna y de la mezcla de solución de hidróxido de sodio y aceitunas durante la etapa de cocido. La viñeta triangular representa la temperatura teórica de la aceituna si sólo se produjera transferencia de calor con el líquido. 
disponible de las aceitunas diluye la solución de $\mathrm{NaOH}$ y con la simplificación que el intercambio de calor entre ambas se produce como si fueran dos soluciones mezcladas, sin tener en cuenta la resistencia a la película ni la de la piel de las aceitunas ni las pérdidas de calor del depósito con el medio exterior, no se podría explicar todo el aumento de calor producido en las aceitunas.

Uno de los posibles responsables del aumento progresivo de temperatura durante la etapa de cocido podría ser el calor generado en las reacciones de hidrólisis alcalina llevadas a cabo por los iones hidroxilo que difunden dentro la aceituna. Estos iones provocan la rotura de los enlaces éster, tanto de la oleuropeína (que es el objetivo del tratamiento) como de los polisacáridos estructurales (pectina, hemicelulosa y celulosa) (Marsilio et al., 1996).

La hidrólisis alcalina de un éster libera calor, es exotérmica (Schneider y Stoessel, 2005; Tjahjono et al., 2009; Taylor y Kluger, 1992; Wadsö, 1958), de modo que las reacciones que se producen dentro de la aceituna generan calor y pueden ser responsables, junto con la transferencia de calor de las aceitunas a la solución y con la dilución de la concentración de sosa, del aumento de temperatura progresivo durante la etapa de cocido.

Al no encontrarse tabulada la entalpía de la reacción de hidrólisis de oleuropeína y sin disponer de la cantidad de cada compuesto que reacciona con los iones hidroxilo, no es posible cuantificar teóricamente el aumento de temperatura que conlleva el conjunto de estas reacciones de hidrólisis alcalina. Así pues, sólo podemos saber cualitativamente que estas reacciones desprenden calor dentro la aceituna y que éste puede ser transmitido a la solución de hidróxido de sodio exterior a través de un proceso de transferencia de energía.

Según los datos experimentales de temperatura dentro la aceituna durante la etapa de cocido, se observa un aumento aproximadamente a partir de los 100 minutos del tratamiento (Figura 8). Este aumento indica que se genera calor dentro la aceituna ya que no puede ser resultado de la transferencia de calor de la solución de $\mathrm{NaOH}$ que se encuentra a una temperatura significativamente inferior. La hipótesis expuesta para explicar este aumento es que el calor se desprende de las reacciones de hidrólisis alcalina y puede que, en menor grado, la dilución del hidróxido de sodio haga también una pequeña aportación. Hay que tener en cuenta que no se han considerado las pérdidas de calor del depósito con el entorno y que por este motivo, probablemente, la generación de calor dentro la aceituna sería aún mayor.

\section{DISCUSIÓN}

Experimentalmente se ha podido determinar el aumento de temperatura de la solución de cocido y el aumento de temperatura de las aceitunas para el estudio de la transmisión de calor. Así, se observa un aumento progresivo y lineal de la temperatura de la mezcla durante la etapa de cocido.

La temperatura de la mezcla de solución de hidróxido de sodio y aceitunas al inicio del tratamiento de cocido influye en otras de las características del proceso. Empezar la etapa de cocido a una temperatura baja, para una misma concentración de sosa, provoca un alargamiento del tiempo necesario para alcanzar el mismo grado de cocido y que la pendiente del aumento lineal de temperatura durante la etapa sea menor. Del mismo modo, los tratamientos que se inician a una temperatura más elevada reducen su duración al ser más rápida la difusión de la sosa (hay más choques intermoleculares) y también consiguiendo un incremento de temperatura ligeramente superior.

La medida de la temperatura interna de las aceitunas evidencia un aumento de temperatura en éstas a partir de un cierto punto del tratamiento de cocido. Parece ser que este aumento resulta de la generación de calor dentro la aceituna dado que por transmisión de calor no se podrían alcanzar estos valores al encontrarse el líquido a una temperatura inferior a la de las aceitunas. La hipótesis expuesta para explicar esta generación es que el calor se desprende de las reacciones de hidrólisis alcalina y puede que, en menor grado, de la dilución del hidróxido de sodio. Hay que tener en cuenta que no se han considerado las pérdidas de calor del depósito con el entorno y que, probablemente, la generación de calor sería aún mayor.

\section{CONCLUSIONES}

A partir de la modelización expuesta en este trabajo, se pueden hacer predicciones de los valores de las variables que describen la transferencia de calor en el proceso de cocido, como la temperatura inicial de la solución de hidróxido de sodio necesaria para finalizar el proceso en un tiempo determinado. Un mayor conocimiento de las variables estudiadas y su aplicación industrial permitirían optimizar el tratamiento de cocido.

\section{AGRADECIMIENTOS}

Los autores quieren agradecer a Nuestra Señora de las Virtudes S.C.A. (www.soberbio.com) y a Víctor Giordana, S.A. División Olivos (www.vgagronegocios.com) la ayuda prestada en el desarrollo de este trabajo.

\section{REFERENCIAS}

Al-Widyan MI, Rababah TM, Mayyas A, Al-Shbool M, Yang W. 2010. Geometrical, thermal and mechanical properties of olive fruits. J. Food Process Eng. 33, 257-271.

Chammem N, Kachouri M, Mejri M, Peres C, Boudabous A, Hamdi M. 2005. Combined effect of alkali pretreatment and sodium chloride addition on the 
olive fermentation process. Bioresour Technol. 96, 1311-1316.

Costa J, Cervera S, Cunill F, Esplugas S, Mans C, Mata J. 2002. Curso de Ingeniería Química. Editorial Reverté, S.A., Barcelona.

Fernández-Díez MJ, de Castro R, Garrido A, GonzálezCancho F, González-Pellissó F, Nosti M. Heredia A, Mosquera IM, Rejano L, Durán MC, Sánchez F, García P, de Castro A. 1985. Biotecnología de la aceituna de mesa. Publicaciones CSIC, Sevilla, Madrid.

Jaramillo Carmona S, de Castro A, Rejano Navarro L. 2011. Proceso tradicional de aderezo de aceitunas verdes de mesa. Racionalización del cocido. Grasas Aceites 62, 375-382.

Kader, AA. 2011. Postharvest technology of horticultural crops. Publication 3529 PDF Third Edition, ANR Publications, University of California, California.

Levenspiel, O. 1998. Flujo de fluidos e intercambio de calor. Editorial Reverté S.A., Barcelona.

Mafra I, Lanza B, Reisa A, Marsilio V, Campestre C, De Angelis M, Coimbra MA. 2001. Effect of ripening on texture, microstructure and cell wall polysaccharide composition of olive fruit (Olea europaea). Physiol. Plant 111, 439-447.

Maldonado MB, Zuritz CA, Assof MV. 2008. Diffusion of glucose and sodium chloride in green olives during curing as affected by lye treatment. J. Food Eng. 84, 224-230.

Maldonado MB, Zuritz CA, Gascón AD, Rey E. 2003. Difusión de sodio en aceitunas verdes durante el tratamiento alcalino. I: Efecto de la concentración de la lejía. Grasas Aceites 54, 358-364.

Maldonado MB, Zuritz CA, Gascón AD. 2003. Difusión de sodio en aceitunas verdes durante el tratamiento alcalino. II: Efecto de la temperatura de la lejía. Grasas Aceites 54, 365-370.

Maldonado MB, Zuritz CA, Wuilloud RG, Bageta CR, Terreni J, Sánchez MJ. 2011. A simple model of the diffusion phenomena taking place during the debittering process of green table olives. Grasas Aceites 62, 39-48.

Maldonado MB, Zuritz CA. 2003a. A model for diffusion of sodium in green olives at different temperatures and lye concentrations. J. Food Process Eng. 26, 339-356.
Maldonado MB, Zuritz CA. 2003b. Modelación matemática del proceso de tratamiento con hidróxido sódico de aceitunas verdes de mesa. Grasas Aceites 54, 180187.

Maldonado MB, Zuritz CA. 2004a. Determination of variable diffusion of sodium during debittering of green olives. J. Food Process Eng. 27, 345-358.

Maldonado MB, Zuritz CA. 2004b. Difusión de sodio durante el tratamiento alcalino de aceitunas variedad aloreña. Grasas Aceites 55, 409-414.

Marsilio V, Lanzaf. B, De Angelis M. 1996. Olive Cell Wall Components: Physical and Biochemical Changes during Processing. J. Sci. Food Agric. 70, 35-43.

Rejano Navarro L, Sánchez-Gómez AH, Vega Macías V. 2008. Nuevas tendencias en el tratamiento alcalino "cocido» de las aceitunas verdes aderezadas al estilo español o sevillano. Grasas Aceites 59, 197-204.

Sánchez Gómez AH, García García P, Rejano Navarro L. 2006. Trends in table olive production. Grasas Aceites 57, 86-94.

Schneider MA, Stoessel F. 2005. Determination of the kinetic parameters of fast exothermal reactions using a novel microreactor-based calorimeter. Chem. Eng. J.115, 73-83.

Solvay Chemicals International. 2004. $\mathrm{NaOH}$ Product Characteristics.

Taylor SD, Kluger R. 1992. Heats of Reaction of Cyclic and Acyclic Phosphate and Phosphonate Esters. "Strain Discrepancy" and Steric Retardation. J. Am. Chem. Soc .114, 3067-3071.

The Dow Chemical Company. 2010. Caustic Soda Solution Handbook.

Tjahjono M, Widjaja E, Garland M. 2009. Combined Online Spectroscopic, Calorimetric, and Chemometric Analysis: Reaction Enthalpy Determinations in Single and Parallel Reactions. Chem.Phys. Chem. 10, 12741283.

Wadsö I. 1958. The heats of hydrolysis of some alkyl acetates. Acta Chem. Scand. 12, 630-634.

Zuritz CA, Maldonado MB. 2004. A simple method to determine diffusion of sodium in the epidermis of green olives. J. Food Process Eng. 27, 328-344.

Recibido: $10 / 1 / 13$ Aceptado: 5/4/13 